\title{
A Novel Static Sector Capacity Evaluation Method and Correlation Study
}

\author{
Sen Du ${ }^{1}$, Jiawei Kang ${ }^{1+}$, Yuqing Ye ${ }^{1}$, Jing Huang ${ }^{1}$, and Zhiyuan Shen ${ }^{1}$ \\ ${ }^{1}$ College of Civil Aviation, Nanjing University of Aeronautics and Astronautics, Nanjing 210016, China
}

\begin{abstract}
At present, there is no clear definition and quantitative standard of static sector capacity for air traffic control, which leads to an unsafe phenomenon that the number of aircraft far exceeds the static sector capacity. Furthermore, economic losses and safety issues are brought when the static capacity is exceeded, but the dynamic capacity is not reached. This paper builds a novel calculation model of static capacity with the proposed concept of Operational Complexity. Then the relationship between Static Sector and Dynamic Sector Capacity is established based on the numerical result of Operational Complexity. Finally, a case study shows the assessment procedure of the proposed correlation for the air traffic control sector.
\end{abstract}

Keywords: sector capacity; dynamic sector capacity; controller load; operational complexity; correlation;

\section{Introduction}

With the development of civil aviation and related industries in leaps and bounds in recent decades, airplanes have gradually become the main transportation for people traveling long distances. Nowadays, under the background of the normalization of the epidemic, the development direction of "domestic circulation as the main body, domestic and international double circulation to promote" makes domestic air routes gradually prominent. However, the conflict between airspace resources and air traffic flow in China is becoming more and more apparent. At the same time, the current research mainly focuses on the accumulated number of aircraft in one duration as a criterion to define the sector capacity, which ignores the Static Sector Capacity. The phenomenon will induce an imbalance of capacity assessment in the sector. Thus the controllers in these sectors have been overloaded for a long time. A new Static Sector Capacity assessment method that can improve the security in air traffic is urgently needed, and the correlation between Static Sector Capacity and Dynamic Sector Capacity is essential to be found out.

Currently, there are four main methods for sector capacity assessment [1]: First, by simulation from computer model; Second, by the analysis of historical data; Third, by building mathematical models; And fourth, by controller' workload.

In the case of computer simulation, although the required data can be obtained immediately after the model has been simulated, this model considered most of the problems from a scant perspective, without considering the differences in each sector. The reference claims that [2], the capacity of the route is divided into two parts: the capacity of the starting point of the route and the fixed capacity of the route itself, and a new maximum capacity model is established so that the length of the route becomes one of the influencing factors of the capacity. At the same time, the dynamic capacity model of the route is established by introducing the influence coefficients of dynamic factors such as real-time weather conditions and airspace activities. The disadvantage of this method is that the model establishment requires more investment and a long evaluation period.

In terms of the analysis based on the historical data, the advantage is that this analysis considers the uniqueness of different sectors. Still, it is difficult to obtain a large amount of data and process them effectively.

Corresponding author. Tel.: +86-15615883881; fax: +025-84893461.

E-mail address: 380978016@qq.com. 
In reference [3], a method for assessing the historical peak capacity of airports was proposed, and the results of this assessment were verified by using a combination of multiple indicators, showing that such an accurate estimate can provide a better benchmark for airport capacity forecasting. The disadvantages of this method are the long assessment time and the difficulty of data processing.

Regarding mathematical computation model evaluation, reference [4] proposed a model for airspace utilization calculation to help airspace managers effectively evaluate airspace utilization. A new definition of airspace utilization is introduced by presenting the "Airspace Load" concept to quantify airspace resources. The reference [5] analyzed the auto-correlation between the number of aircraft in a sector and the controller's workload while proposed a sector capacity assessment method based on the Exponential Smoothing Model, the feasibility of this method was verified by using the data which come from a sector in the northwest of China. The disadvantage of this method is that the model is biased, and the influence factors on sector capacity are not fully considered.

Finally, the method based on controllers' workload is the best assessment method currently. However, most of the processes in this method only use controllers' workload hours as a measure to evaluate capacity. Still, the calculation method provided by ICAO defines controller workload as Communication Load, Thinking Load, and Operation Load, and the Total Load is obtained by adding up the time of these three. In addition, existing studies perform a simple linear analysis of controllers' load, which without considering the cumulative nature of controllers' workload. In reference [6], a new controllers' workload assessment method is proposed by using a more straightforward regression analysis with specific airspace as an example. In the reference [7], a simple hierarchical treatment of controllers' workload was carried out, and a linear model of the load was established by combining the data. In terms of the controller's voice, the reference [8] takes the tower controllers' Voice Load as the research object, analyses the controllers' voice by using MATLAB, performs factor analysis by using SPSS software, and characterizes the Voice Load by using the extracted characteristic parameters, after that, a functional relationship between the controllers' workload and the number of aircraft is established. This method manipulates the Voice Load but does not consider the specific content in controllers' instructions. The reference [9], taking the Xi'an terminal area as an example, considers the influence of airspace complexity on controllers' workload and constructs an airspace complexity calculation model. Using DOROTASK, a controllers' workload quantification method, based on the dynamic weighting of airspace complexity, is proposed. This method quantifies and analyses the controllers' workload.

It can be seen that the above methods are all Dynamic Sector Capacity assessment methods. There is still a gap in the Static Sector Capacity assessment research, which is significant to the study of Air Traffic Flow. Also, the safety and economic benefits of Air Traffic Management are huge; Therefore, it is essential to establish an assessment system, which could combine both dynamic and static capacity as the standard so that the controllers' workload and Sector Capacity can be assessed scientifically and effectively.

The remainder of this paper is organized as follows: Section 2 briefly introduces the concept of Operational Complexity, Dynamic Sector Capacity, and Static Sector Capacity, Section 3 describes the correlation between Dynamic Sector Capacity and Static Sector Capacity, Section 4 presents the conclusions, and acknowledgment is drawn in Section 5.

\section{Operational Complexity}

\subsection{Operational Complexity and Average Operational Complexity}

To establish the relationship between Dynamic Sector Capacity (DSC) and Static Sector Capacity (SSC), this paper introduces the concept of Operational Complexity (OC), that is, the instantaneous workload of controllers; and the idea of Total Operational Complexity (TOC): the total workload of controllers during the load duration. The OC is subject to multiple factors, encompassing the objective elements of airspace, the subjective factors of controllers, and many other factors [10].

Based on the definition of Dynamic Sector Capacity mentioned above, and the controller's operation habits in daily work, while considering above subjected factors, this paper proposes the numerical illustration of Average Operational Complexity (AOC): In the time range-- i (seconds) to $\mathrm{r}$ (seconds), $\mathrm{Xi}$ are the 
algebraic values of $\mathrm{n}$ operation instructions which are performed by the controllers and multiplied by the weighting factor of the operation instructions, finally divided by the total time interval $i+r$ (seconds) to get the AOC in this time interval, i.e.

$$
A O C=\frac{\sum_{i=0}^{i=n} \theta i * X i}{i+r}
$$

In Eq. (1), $\theta \mathrm{i}$ is the weighting factor of each operation, and different operations have different weighting factors, these factors will be obtained according to the further experiments. $\mathrm{Xi}$ is the algebra of operation, which includes: (1) Additional instruction X1, contains conflict deployment, the change of en-route, the execution of waiting procedures and other instructions; (2) Basic instruction X2: greetings, speed adjustment, the deployment of altitude, steering and other instructions; (3) Other operations X3: filling the sheet, screen operations.

\subsection{Dynamic Sector Capacity}

The relationship between the TOC and the AOC is

$$
T O C=\int A O C d t
$$

However, Eq. (2) does not consider the additional workload, which comes from continued work, the controller's Waiting Operation, which comes from waiting time, and Surveillance Load, which stems from the situation that the controllers have to monitor other aircraft in this sector which are not be controlled by controllers at that time; Therefore, it is necessary to add a correction parameter Wy to Eq. (2), which represents several extra operational workloads, so as to obtain the complete formula for the Total Operational Complexity:

$$
T O C=\int A O C d t+W_{y}
$$

According to the definition of DSC mentioned above, it is easy to obtain the maximum TOC in one sector depend on historical date. When the TOC of controllers in the working duration reaches $70 \%$ of $\mathrm{TOC}_{\max }$ in this sector, the number of aircraft are flying in this sector is the DSC in this sector.

\subsection{Static Sector Capacity}

According to the definition of Dynamic Sector Capacity, while combining the actual operation date in each sector, the definition of Static Sector Capacity is that the number of aircraft the controllers could control instantaneously.

Also, the AOC is taken as the limit to obtain the Instantaneous Operational Complexity (IOC) in each sector:

$$
I O C=\lim _{t \rightarrow 0} A O C
$$

Based on the above definition, while according to the relevant experiment data and ICAO 9426, which stipulate that the operation limitation in one sector is the time when the workload of controllers is reaching $80 \%$ of the maximum workload in this sector, this paper proposes a provision that: according to the historical data in one sector, the maximum value of IOC in this sector is obtained, after that, when one controller is working in this sector, and the IOC of this controller reaches $80 \%$ of $\mathrm{IOC}_{\max }$, at that time, the number of aircraft are operated by that controller is the SSC of this sector, which means when the Eq. (5) hold, the number of aircraft operated by the controllers is the SSC of this sector.

$$
I O C=I O C_{\max } * 80 \%
$$

\section{Correlation}

\subsection{Schematic Diagram}

This section uses a schematic to explain the correlation between them to establish evaluation criteria that combine dynamic and static sector capacity. 


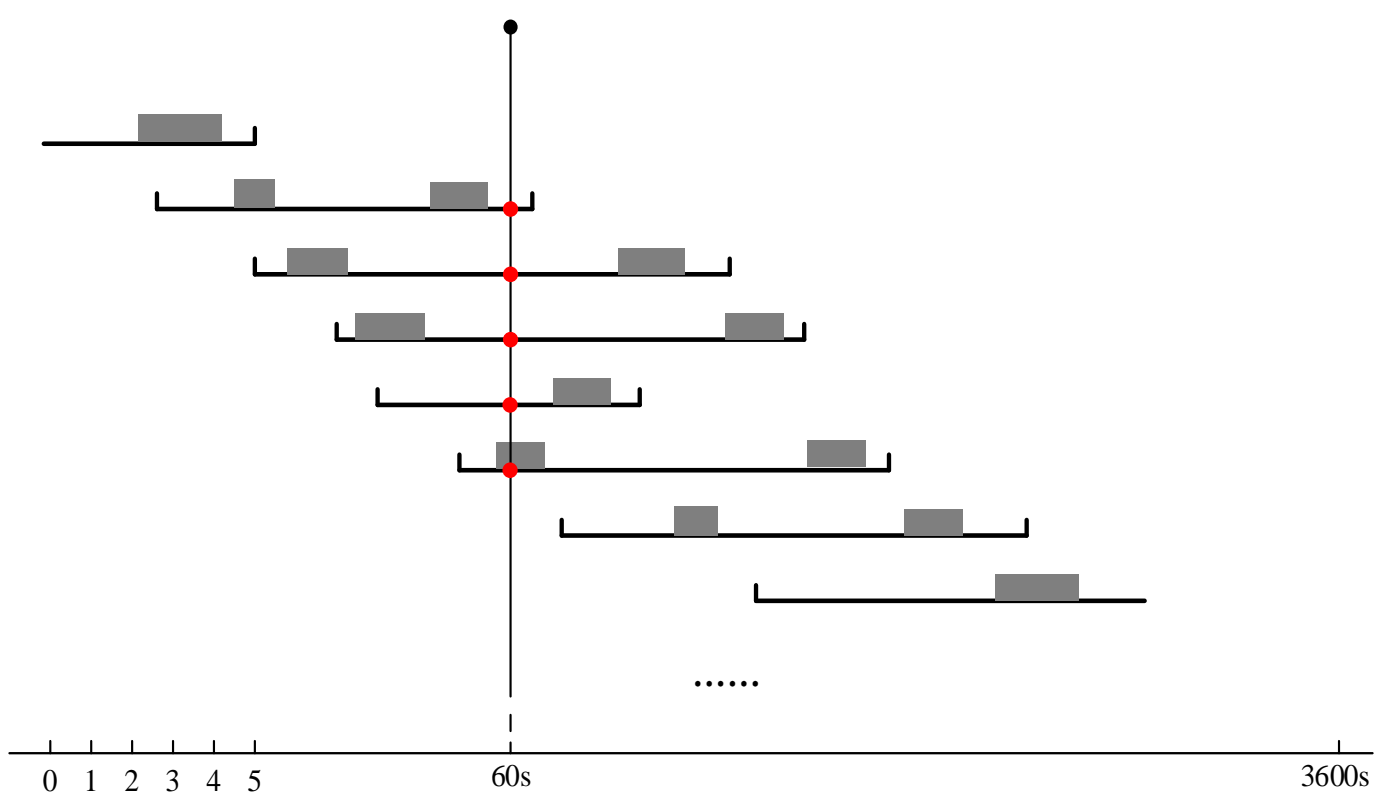

Fig. 1: Time of aircraft in the sector.

In Fig. 1, the horizontal axis represents time, with the standard time interval of 1 hour and the basic unit of 1 second. The black lines in the figure show the time of the aircraft in the sector, arranged in a stepped pattern according to the order in which they enter the sector. According to Fig. 1, the SSC at a given moment can be expressed as the number of intersections between the vertical line, which perpendicular to the horizontal axis, and the line segments.

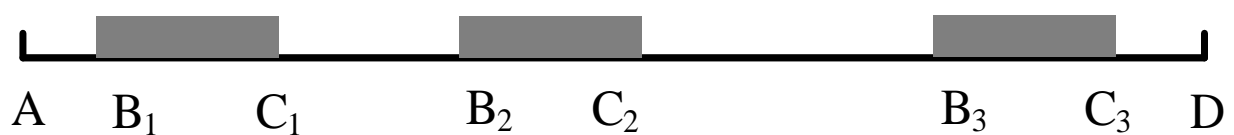

Fig. 2: A black line in Fig. 1.

Fig. 2 shows a line in Fig. 1, where point $A$ and point $D$ are the points at which the aircraft enters and leaves the sector, respectively, and the line $\mathrm{BiCi}$ is the time the controller spends to operate the aircraft $(\mathrm{i}=1,2,3)$. In addition, in practical applications, since the time of aircraft entry and leave in the sector cannot be obtained from the recording, the lines $\mathrm{AB} 1$ and $\mathrm{C} 3 \mathrm{D}$ can be ignored.

When the voice data is obtained, the AOC corresponding to each moment in the black shaded area can be calculated using Eq. (1), and the total load for that hour can be obtained by summing the TOC of all line in that hour by using Eq. (3). The corresponding DSC can be obtained by adjusting. Similarly, using Eq. (4) to calculate the IOC of the intersection points and then adding them together, The SSC can be obtained with adjustment by using Eq. (5).

At the same time, the controller cannot talk to more than one aircraft, so only one black shaded area intersects the line at each time. The operational complexity calculated from the remaining intersection points is only the controller's monitoring load for these aircraft.

\subsection{The process of Capacity Assessment}

This section proposes an evaluation method that combines both SSC and DSC.

Fig. 3 shows the process of sector capacity assessment, after combining dynamic capacity and static capacity criteria, there are the following steps:

- Collect peak hour controller voice data.

- Calculate peak hour AOC, TOC, and IOC.

- Adjust the TOC, IOC according to the sector structure and calculate $\mathrm{TOC}_{\max }$ and $\mathrm{IOC}_{\max }$.

- Calculate the IOC and TOC corresponding to the sector which needs to be evaluated.

- Adjust the SSC and DSC until the criteria are satisfied. 


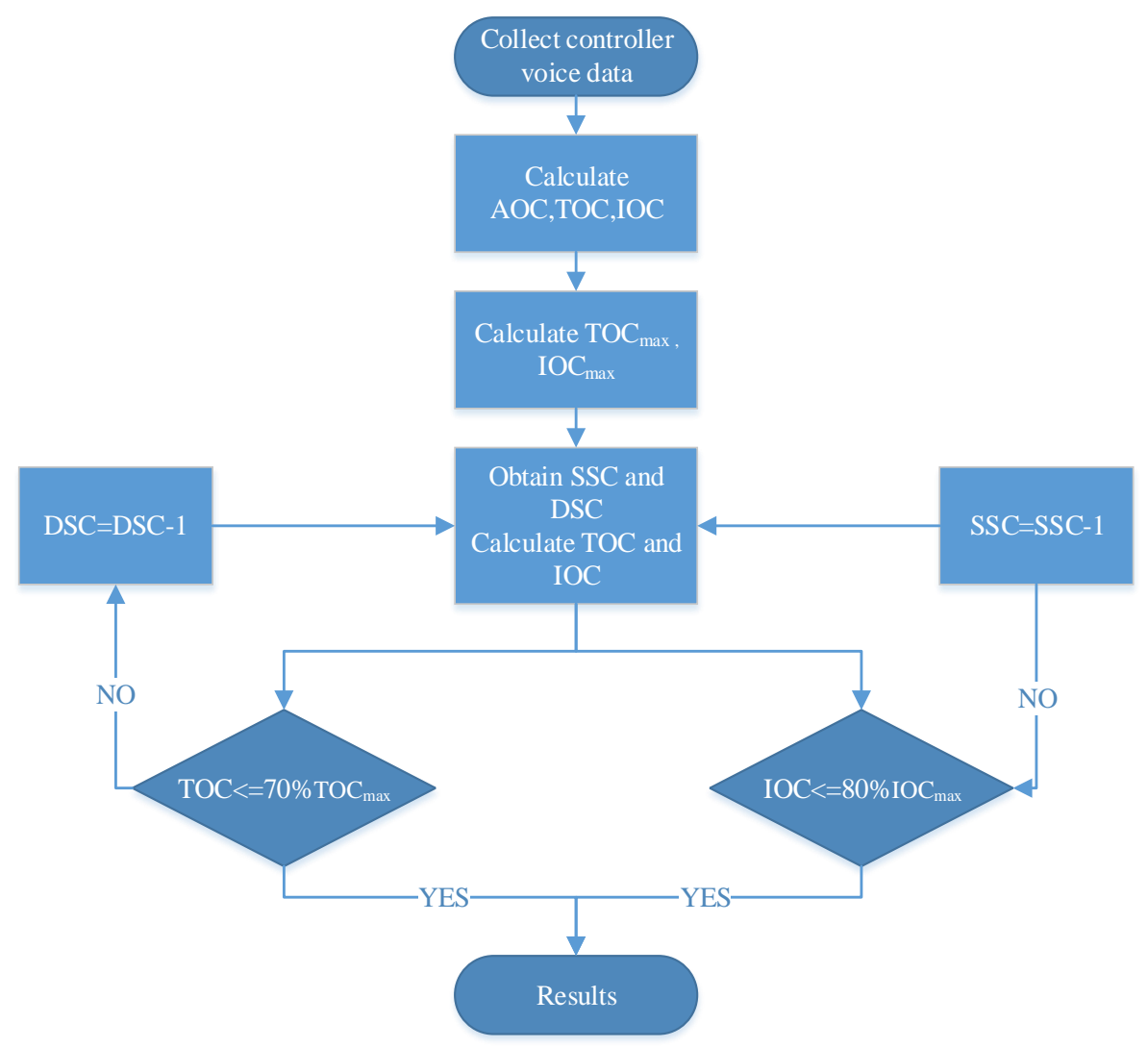

Fig. 3: The flow chart of capacity assessment.

\section{Conclusions}

This paper proposed a novel method to calculate the static capacity of the sector. Furthermore, the relationship between static capacity and dynamic capacity was also built. The proposed model provides a visualization figure explanation to restrict controllers' workload in each sector for the Civil Aviation Administration of China. A case study shows the assessment procedure of the proposed correlation for the air traffic control sector. Further research will focus on the experiments with additional radiotelephony data.

\section{Acknowledgment}

The authors would like to thank the financial support by the Open Fund of College Student Innovation, and Entrepreneurship Training Program of Nanjing University of Aeronautics and Astronautics funded project (202110287054Z).

\section{References}

[1] Zhou X, Hu M . An Overview on Airspace Capacity Evaluation. Journal of Civil Aviation Flight University of China, 2016.

[2] Jing Y U , Liu H, Xiong Y Y, et al. An improved evaluation model of En-route dynamic capacity[J]. Journal of Sichuan University(Natural Science Edition), 2007.

[3] Zheng Zhao. Research on Airspace Capacity Evaluation and Forecast Technology [D].Nanjing University of Aeronautics and Astronautics,2015.

[4] Zhaoning Zhang,Ping Wang. Computing Model of Airspace Utilization Rate Based on Airspace Load[J]. Journal of Networks,2014,9(1).

[5] Ren G J , Zhu J F, Chao-Yang L U . Research on Assessment Method of Sector Capacity Based on ES Model. Aeronautical Computing Technique, 2018.

[6] Pei C, Han S , Liu X . Regression analysis method for evaluating ATC controller workload. Journal of Nanjing University of Aeronautics \& Astronautics, 2007.

[7] Sifra Christina Corver,Dana Unger,Gudela Grote. Predicting Air Traffic Controller Workload[J]. Human Factors: 
The Journal of Human Factors and Ergonomics Society,2016,58(4).

[8] Qiong Zhang.Research on Capacity Evaluation Based on Control Voice Load[J].Technology Innovation and Application,2021(02):26-30.

[9] Lulu Ling. Research on Xi'an Terminal Sector Dynamic Optimization Base on Controller Workload[D].Civil Aviation Flight University of China,2018.

[10] Yong Tian, Lili Wan and Shuangshuang Yang, "Research on sector dynamic capacity evaluation," 2014 Integrated Communications, Navigation and Surveillance Conference (ICNS) Conference Proceedings, Herndon, VA, USA, 2014, pp. J3-1-J3-8, DOI: 10.1109/ICNSurv.2014.6819995. 